\title{
Cerebellar Contributions to Reach Adaptation and Learning Sensory Consequences of Action
}

\author{
Jun Izawa, Sarah E. Criscimagna-Hemminger, and Reza Shadmehr \\ Laboratory for Computational Motor Control, Department of Biomedical Engineering, Johns Hopkins School of Medicine, Baltimore, Maryland 21205
}

When we use a novel tool, the motor commands may not produce the expected outcome. In healthy individuals, with practice the brain learns to alter the motor commands. This change depends critically on the cerebellum as damage to this structure impairs adaptation. However, it is unclear precisely what the cerebellum contributes to the process of adaptation in human motor learning. Is the cerebellum crucial for learning to associate motor commands with novel sensory consequences, called forward model, or is the cerebellum important for learning to associate sensory goals with novel motor commands, called inverse model? Here, we compared performance of cerebellar patients and healthy controls in a reaching task with a gradual perturbation schedule. This schedule allowed both groups to adapt their motor commands. Following training, we measured two kinds of behavior: in one case, people were presented with reach targets near the direction in which they had trained. The resulting generalization patterns of patients and controls were similar, suggesting comparable inverse models. In the second case, participants reached without a target and reported the location of their hand. In controls, the pattern of change in reported hand location was consistent with simulation results of a forward model that had learned to associate motor commands with new sensory consequences. In patients, this change was significantly smaller. Therefore, in our sample of patients, we observed that while adaptation of motor commands can take place despite cerebellar damage, cerebellar integrity appears critical for learning to predict visual sensory consequences of motor commands.

\section{Introduction}

It is thought that when the brain generates a motor command, it also predicts the sensory consequences (Körding and Wolpert, 2004; Vaziri et al., 2006; Shadmehr et al., 2010). This association of motor commands with the sensory consequences is called a forward model. The neural system that forms this association is unknown, though there is some evidence that for arm movements the cerebellum may play a critical role: developmental damage (Nowak et al., 2007) or temporary disruption (Miall et al., 2007) of the cerebellum produces behavioral deficits that suggest an inability to accurately predict sensory consequences of motor commands. However, there is also evidence against this argument. For example, cerebellar patients are generally unimpaired in adjusting the left hand predictably when they use the right hand to pick up an object that is resting on the left hand (Diedrichsen et al., 2005). Does this imply that the forward model is unaffected by cerebellar damage? Or does it imply that with cerebellar damage, in certain tasks, there is a latent ability to produce approximately the correct motor commands, despite being unable to predict their sensory consequences?

Received Dec. 21, 2011; revised Feb. 5, 2012; accepted Feb. 10, 2012.

Author contributions: J.I., S.E.C.-H., and R.S. designed research; J.I., S.E.C.-H., and R.S. performed research; J.I. and R.S. contributed unpublished reagents/analytic tools; J.I. analyzed data; J.I. and R.S. wrote the paper.

This work was supported by NIH Grants NS057814, the Human Frontiers Science Foundation, and Japan Society for the Promotion of Science (Grants-in-Aid for Scientific Research 23700360). We are grateful for comments of Dr. Maurice A. Smith, who suggested the idea that adaptation of the forward model may leave its signature in the generalization patterns of the inverse model. We are also grateful for the comments of Dr. Amy Bastian.

Correspondence should be addressed to Jun Izawa, ATR Computational Neuroscience Laboratories, 2-2-2 Hikaridai, Keihanna Science City, Kyoto 619-0288, Japan. E-mail: jizawa@atr.jp.

DOI:10.1523/JNEUROSCI.6353-11.2012

Copyright $\odot 2012$ the authors $\quad 0270-6474 / 12 / 324230-10 \$ 15.00 / 0$
Here we present an experiment in which we attempt to disentangle the process of learning to produce the correct motor commands (i.e., commands that improve performance) from the process of learning to predict the sensory consequences of those commands. The process of learning to produce the correct motor commands is typically labeled as learning an inverse model. An inverse model associates a goal with the motor commands that are successful in achieving the goal (Kawano et al., 1996; Kawato, 1999). Some have hypothesized that both the inverse and forward models reside in the cerebellum (Wolpert and Kawato, 1998; Haruno et al., 2001). If this is true, then cerebellar damage might produce both an inability to learn the correct motor commands (Maschke et al., 2004; Smith and Shadmehr, 2005; Rabe et al., 2009) and an inability to learn the sensory consequences of those commands (Synofzik et al., 2008). In contrast, suppose that the cerebellum is only associated with learning of forward models. Furthermore, suppose that forward models are a sufficient but not a necessary condition for learning to produce the motor commands that compensate for a perturbation (Izawa and Shadmehr, 2011). If these two assumptions are valid, then, at least in some cases, cerebellar damage should only impair the ability to learn the sensory consequences of motor commands, while sparing the ability to learn the correct motor commands.

To dissociate these two possibilities, we rely on a recent finding: when a perturbation is applied gradually, people with cerebellar damage have the ability to improve their motor commands, producing approximately the correct motor output (CriscimagnaHemminger et al., 2010). Here, we rely on gradual perturbations to dissociate between the process that learns to improve the motor commands and the process that learns to predict the sensory consequences of those commands. 
Table 1. Characteristics of patients with cerebellar degeneration

\begin{tabular}{llllll}
\hline Identifier & Gender & Age (years) & Handedness & Diagnosis & ICARS \\
\hline 1 & M & 37 & $\mathrm{~L}$ & SCA8 & 46 \\
2 & $\mathrm{M}$ & 54 & $\mathrm{R}$ & SCA6 and SCA8 & 63 \\
3 & $\mathrm{~F}$ & 67 & $\mathrm{R}$ & Autosomal dominant & 55 \\
4 & $\mathrm{~F}$ & 67 & $\mathrm{R}$ & SCA6 & 5 \\
5 & $\mathrm{M}$ & 75 & $\mathrm{R}$ & R PICA and R SCA stroke & 17 \\
6 & $\mathrm{~F}$ & 65 & $\mathrm{R}$ & Sporadic & 35 \\
7 & $\mathrm{M}$ & 47 & $\mathrm{R}$ & Sporadic & 51 \\
8 & $\mathrm{~F}$ & 57 & $\mathrm{R}$ & SCA6 & 33 \\
9 & $\mathrm{~F}$ & 52 & $\mathrm{R}$ & SCA6 & 23 \\
\hline
\end{tabular}

ADCA, autosomal dominant ataxia.

\section{Materials and Methods}

Nine individuals with cerebellar ataxia (Table 1) and 10 neurologically healthy age-matched control subjects ( 6 males, 4 females) participated in this study. The mean age of the patients was $57.8 \pm 3.9$ years and that of the control subjects was $63.1 \pm 1.43$ years.

Three of our patients were diagnosed with spinocerebellar ataxia (SCA) type 6, one patient had both SCA6 and SCA8, one had SCA8, one had autosomal dominant cerebellar disease (in which clinical symptoms of ataxia tend to manifest in mid-adulthood), and one subject had a stroke affecting the right posterior inferior cerebellar artery (PICA) and SCA territories of the cerebellum. The other patients had either sporadic adult late-onset ataxia or autosomal dominant cerebellar ataxia (i.e., pure cerebellar syndrome with unknown genetics).

The severity of ataxia was rated using the International Cooperative Ataxia Rating Scale (ICARS) (Trouillas et al., 1997). This scale is from 0 to 100 , with the larger values indicating greater disability. Clinical examination showed no evidence of hypertonia, sensory loss (proprioception and fine touch via monofilament), or extrapyramidal features in the arms of these individuals. Experimental procedures were approved by the Johns Hopkins University School of Medicine Institutional Review Board and all subjects signed a consent form.

Task. In a darkened room, subjects sat in front of a robotic arm and held its handle with their right arm. The robotic arm was built in 1994 and is controlled with hardware and software that were designed and built by scientists in the Shadmehr Lab. The subjects were asked to reach toward a target that was projected onto a screen directly above the plane of motion (Fig. 1A) (Izawa and Shadmehr, 2008). The white screen covered the arm, preventing direct view of the hand. The experimental session was composed of three consecutive phases: baseline, adaptation, and postadaptation (Fig. $1 B$ ). During a reaching movement, position data were recorded at sampling frequency of $100 \mathrm{~Hz}$. For analysis of hand trajectories, we used Savitzky-Golay smoothing filter for the measured hand position and velocity.

In the baseline phase, the robot positioned the hand in a start box, at which point a target of $6^{\circ}$ width appeared at a radius of $10 \mathrm{~cm}$ and angle of $45^{\circ}$. Subjects were instructed to perform a shooting motion so that their hand crossed within the target area and punched a virtual pillow placed beyond the target (the pillow was simulated by the robot). Thus, the robot assisted with stopping of the movement. We have found this design is useful in increasing the comfort of cerebellar patients (Tseng et al., 2007; Criscimagna-Hemminger et al., 2010), as it eliminates the tremor that they typically exhibit when they are attempting to stop at the target. In the first block of the shooting task (50 trials), the cursor was projected on top of the handle. There was no disturbance between the cursor and the actual hand position. This was followed by 25 trials without the cursor projection, i.e., subjects reached towards the visual target without seeing the cursor representing their hand.

Subsequently, subjects performed a localization task, modeled after Synofzik et al. (2008). In the localization task, no targets or cursors were displayed. Rather, a circle with a $10 \mathrm{~cm}$ radius positioned at the center hand location was displayed and the subject made a movement with their right hand to anywhere within the first quadrant $\left(0-90^{\circ}\right)$ without visual feedback. Once the robot brought the hand back to the center, the subject pointed with their left hand to where they believed their right hand crossed the circle (the view of their left hand was unobstructed). That is, the subjects were asked to estimate the location of their right hand at the end of the previous movement. To record the motion of the left hand, we used a Mimio stylus pen.

This combination of shooting with the right hand and localization with the left hand was repeated 25 times in the localization task. Our design of the localization task aimed to minimize the influence of proprioception from the right arm (by holding it at center, rather than holding it at end of the movement). In this way, we hoped to assay the subjects' estimate of where their hand had been as a consequence of the motor commands during the shooting movement, rather than the proprioceptive feedback if the hand were to be held there.

Finally, subjects participated in a set of generalization trials (50 trials). In these trials, a target was presented and subjects made a shooting movement towards that target. The position of the target was selected randomly from $\left[15^{\circ}, 25^{\circ}, 35^{\circ}, 45^{\circ}, 55^{\circ}, 65^{\circ}, 75^{\circ}\right]$. The frequency of the $45^{\circ}$ target was 20/50 trials and that of each peripheral target was $5 / 50$. Cursor feedback was provided for the $45^{\circ}$ target but not to the other targets. The objective of the peripheral targets was to test generalization.

The localization task required subjects to remember the location of their right hand in the previous movement and to point to that location with their left hand. To check that the controls could maintain location of a sensory stimulus in working memory, in the baseline phase we included a task in which subjects were presented a target, waited for $1 \mathrm{~s}$ after the target disappeared, and then pointed with their left hand to the remembered target location.

The baseline phase was followed by a gradual adaptation phase in which subjects experienced zero rotation in the first 20 trials and then the perturbation increased by $5^{\circ}$ every 20 trials until it reached $15^{\circ}$ (Fig. $1 \mathrm{~B}$ ). After a short break, the adaptation task was started with $15^{\circ}$ rotation and increased again by $5^{\circ}$ every 20 trials until it reached $30^{\circ}$. After a second short break, subjects experienced 60 additional trials with the $30^{\circ}$ perturbation and then were tested in the postadaptation phase. During this phase, the target was always at $45^{\circ}$.

In the postadaptation phase, the subjects were tested for the generalization of the adaptation. The target position was selected randomly from $\left[15^{\circ}, 25^{\circ}, 35^{\circ}, 45^{\circ}, 55^{\circ}, 65^{\circ}, 75^{\circ}\right]$. The frequency of the trained target $\left(45^{\circ}\right)$ was $107 / 137$ and that of each peripheral target was $5 / 137$. After a short break, subjects experienced 60 additional trials with the $30^{\circ}$ perturbation and then were tested in the localization task.

Analysis. In the shooting and the localization tasks, our performance measure was the endpoint angle of the reach. In the localization task, our performance measure was the angular distance between the actual endpoint angle of the hand and the angle indicated by the subject. We expected that after adaptation to the visuomotor perturbation, the healthy subjects would alter their belief regarding the sensory consequences of their motor commands. This change was assessed as the within-subject difference in localization from the baseline condition to the postadaptation condition.

Estimating the forward model. If adaptation produces a change in the forward model, then the subject should alter his/her belief regarding sensory consequences of the motor commands in the postadaptation phase with respect to baseline. To assess how much this belief had changed, we asked the subjects to make a self-selected motion with their right hand to anywhere along a $90^{\circ}$ arc, and then point with their left hand to where they believed the endpoint of their right hand motion was. Because the self-selected motion could be to any position from $0^{\circ}$ to $90^{\circ}$, in effect they were generalizing their adaptation from the $45^{\circ}$ target to neighboring movements. Furthermore, because they repeatedly made selfgenerated motions without readapting to the $45^{\circ}$ target, we expected some decay of the original learning. Therefore, to compare performance of the two groups of subjects, we first had to fit a function to the performance of each group to represent the spatial and temporal properties of the results of the localization task. We used the following set of equations to characterize the results of the localization task in the postadaptation phase.

$$
\begin{gathered}
\hat{h}=a_{1} \cos \left(a_{2}\left(h-a_{3}\right)\right) \\
a_{1}=\beta_{1} t+\beta_{2} \\
a_{2}=\beta_{3} t+\beta_{4} \\
a_{3}=\beta_{5} t+\beta_{6},
\end{gathered}
$$


where $h$ is the actual reach angle (deg), $t$ is the trial number, $a_{1}$ is associated with the magnitude of the function, $a_{2}$ is associated with the width, and $a_{3}$ is associated with the center. We estimated the best fit parameters, $\beta_{*}$, that minimized the squared error between the model prediction and the actual data. To estimate the confidence interval, we used a bootstrapping method and estimated the parameter set using a nonlinear optimization algorithm (1sqnonlin in Matlab6.5).

Estimating the inverse model. By definition, the inverse model is an association between the goal and the motor commands. To estimate the inverse model, we trained movements to a target at $45^{\circ}$ and then measured the motor commands that the subjects produced to this and neighboring targets. We represented the resulting function as

$$
h=b_{1} \cos \left(b_{2}\left(g-b_{3}\right)\right) \text {, }
$$

where $g$ indicates the goal (target direction). Because the cursor feedback provided for the $45^{\circ}$ target kept the level of the adaptation constant throughout the block of the postadaptation generalization, we assumed that each parameter $b$ was constant throughout the block. We identified the model parameters using the same procedure as described for the forward model.

Modeling the adaptation process. To better understand our behavioral data, we considered a simple computational model of adaptation in which the brain uses the observed sensory feedback to update its belief about the consequences of motor commands, and then uses this updated map to search for motor commands that produce a movement to the goal. In our simulations, when the hand moves at direction $u$, the cursor moves at direction $c$. These two variables are related via perturbation $p$ :

$$
c=u+p .
$$

The forward model encodes the relationship between motor commands $u$ and sensory consequences (cursor position) $c$ using basis elements $g_{\mathrm{i}}$ via a population code:

$$
\begin{gathered}
\hat{p}(u ; w)=w^{\mathrm{T}} g(u) \\
g=\left[g_{1}(u), g_{2}(u), \ldots, g_{\mathrm{m}}(u)\right]^{\mathrm{T}} \\
w=\left[w_{1}, \ldots, w_{\mathrm{m}}\right]^{\mathrm{T}}
\end{gathered}
$$
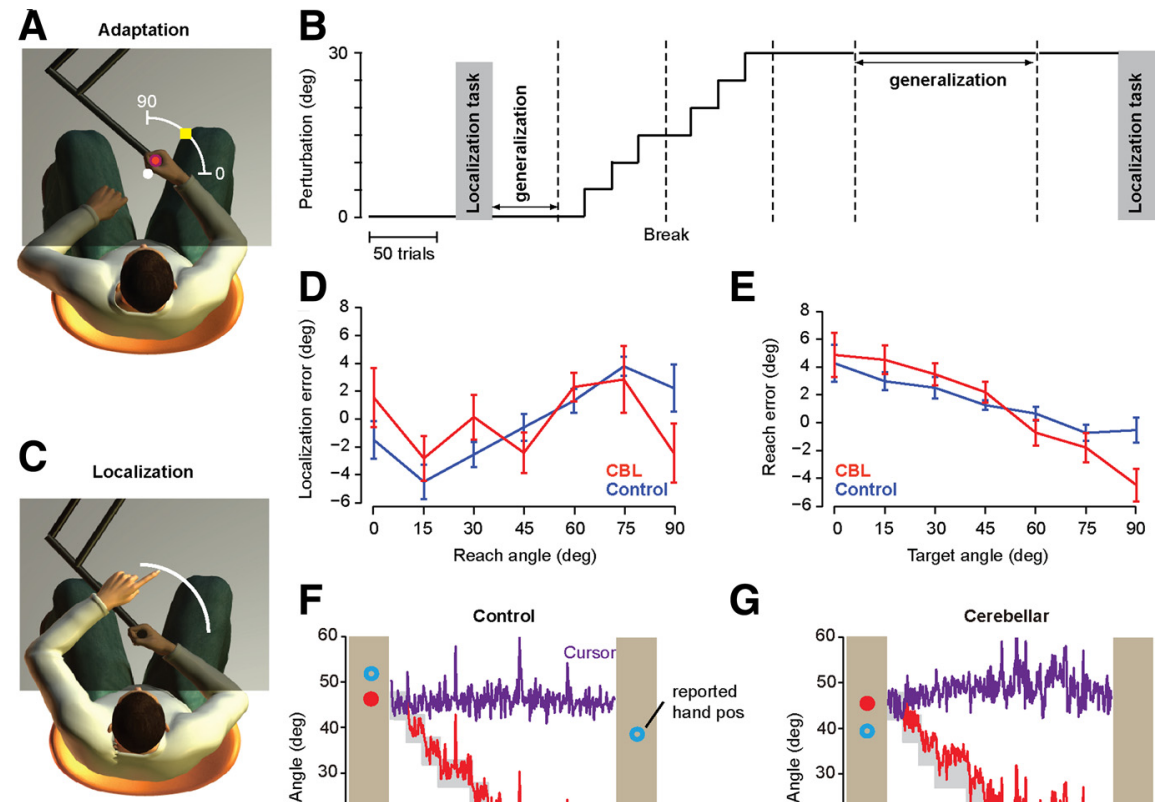

D

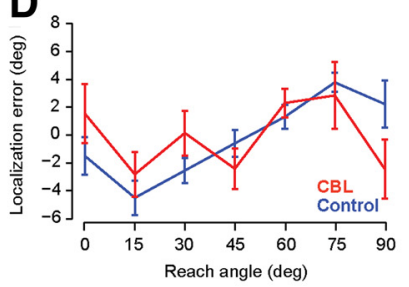

$\mathbf{F}$

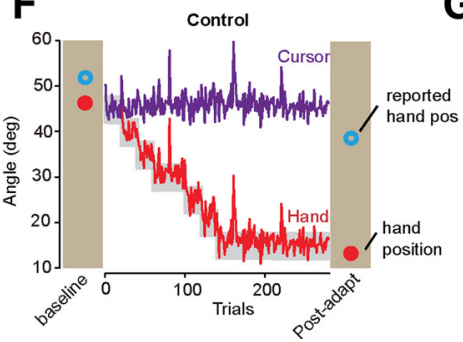

E

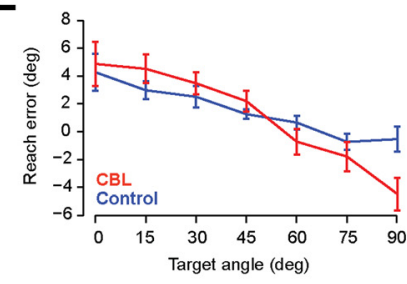

G



Figure 1. Experimental setup and data from representative subjects. $A$, Experimental setup. Subjects held the handle of a robot manipulandum and viewed the image projected on a screen that covered both their limb and the robot. The subjects performed a shooting movement (quick reaching out) to go through a target at $45^{\circ}$ and hit a virtual pillow. The white dot at center indicates their start position. The white arc (radius, $10 \mathrm{~cm}$, centered at the start position) indicates the boundary of the task space. As the hand crossed the arc, the robot produced a virtual pillow and assisted in bringing the hand back to center. During targeted reaching, the arc was not visible. $\boldsymbol{B}$, Time course of the experiment. Localization task is explained in $\boldsymbol{C}$. Generalization task involved reaching to targets other than the trained target at $45^{\circ}$. The adaptation phase includes 220 trials in which a perturbation was gradually imposed on the relationship between hand motion and cursor motion. C, In the localization task, without being given an explicit target, the subjects made a reach to cross the arc without any form of visual feedback. After the robot brought the hand back to the start position, subjects pointed with their left hand to the remembered location of their right hand as it crossed the arc in the previous trial. The arc is displayed only in the localization task. $\boldsymbol{D}$, Baseline performance in the localization task. Localization error is the difference between estimated hand position at the end of the reach and actual hand position. $\boldsymbol{E}$, Baseline performance in the generalization task. Reach error is the difference between reach angle and target angle. The movements were attracted toward the $45^{\circ}$ target, the target for which the movements were repeated. $\boldsymbol{F}$, Data from a representative control subject. The result of a reach in the localization task is shown for the baseline condition. The red filled circle is actual reach angle and the open blue circle is the reported angle (reach percept). During the training phase, a target was displayed at $45^{\circ}$ and a visual rotation was gradually imposed. The gray region represents reach angle for which the cursor would strike the target area. The red trace represents actual reach angle. The three large spikes reflect set breaks. The result of a reach in the postadaptation localization task is shown in the final column. The hand reached toward $15^{\circ}$ but the subject reported the hand at near $40^{\circ}$. $G$, Data from a representative cerebellar subject (CBL). Format is the same as in $\boldsymbol{F}$.

In our simulations, the bases were Gaussian functions with a SD of $25^{\circ}$ and centers at $5^{\circ}$ intervals. Thus, the predicted sensory consequence of motor command $u$ is as follows:

$$
\hat{c}=u+w^{\mathrm{T}} \boldsymbol{g}(u) .
$$

Suppose that on trial $n$, a target is shown at position $c^{\star}$. Using the forward model, we produce the motor command that is expected to minimize the difference between $c^{\star}$ and $\hat{c}$ :

$$
u^{(n)}=\underset{u}{\arg \min }\left(c^{\star}-u-\boldsymbol{w}^{T} \boldsymbol{g}(u)\right)^{2} .
$$

As a consequence of producing command $u^{(\mathrm{n})}$, we observe cursor motion $c^{(\mathrm{n})}$. Using the prediction error $c^{(\mathrm{n})}-\hat{c}^{(\mathrm{n})}$, we update our forward model:

$$
\begin{gathered}
e^{(n)}=\frac{1}{2}\left(c^{(n)}-\hat{c}^{(n)}\right)^{2}=\frac{1}{2}\left(p^{(n)}-\hat{p}^{(n)}\right)^{2} \\
w_{\mathrm{i}}^{(n+1)}=\alpha w_{\mathrm{i}}^{(n)}-\eta \frac{\partial e_{\mathrm{i}}}{\partial w_{\mathrm{i}}}=\alpha w_{\mathrm{i}}^{(n)}+\eta\left(c^{(n)}-\hat{c}^{(n)}\right) g_{\mathrm{i}}\left(u^{(n)}\right) .
\end{gathered}
$$

In our simulations, the learning parameters were set to $\alpha=0.99$ and $\eta=$ 0.1 . We simulated this system of equations for a perturbation schedule that followed the experimental protocol. After training, we assayed the change in the forward model by presenting movements in various directions and quantified the predictions of the model (Eq. 4). Similarly, we assayed the change in the inverse model by presenting targets at various directions and quantified the motor commands produced by Equation 6 . 


\section{Results}

We considered an adaptation task in which people reached and placed a cursor in a target. The relationship between the position of the hand and the cursor underwent a gradual schedule of rotation: the cursor motion was perturbed so that as the hand moved to $45^{\circ}$, the cursor moved to $75^{\circ}$, i.e., a visuomotor rotation that reached a peak of $30^{\circ}$. We chose the gradual form of the perturbation because previously we have found that, whereas cerebellar patients are severely impaired in adapting their reaching movements to an abruptly introduced force perturbation (Smith and Shadmehr, 2005), they were less impaired when that perturbation was imposed gradually (Criscimagna-Hemminger et al., 2010). Here, we wondered whether people with cerebellar damage could adapt their motor commands in response to a gradual visuomotor perturbation, and if so, would this adaptation be accompanied by a change in the perceived sensory consequences of the motor commands.

\section{Performance during the baseline phase}

During the baseline phase, a target was presented at $45^{\circ}$. When visual feedback was provided, the average reach angle across subjects was $45.56 \pm 0.14^{\circ}$ in the control group and $46.08 \pm 0.34^{\circ}$ in the cerebellar group (not significantly different; two-tailed $t$ test, $p=0.16$ ). Without visual feedback, the average reach angle was $46.34 \pm 0.42^{\circ}$ for the control group and $48.52 \pm 1.23^{\circ}$ for the cerebellar group (not significantly different; two-tailed $t$ test, $p=$ 0.21 ). Therefore, there was no systematic difference in the bias of the reaching movements between groups. However, the cerebellar group showed larger variability than the control group when the visual feedback was provided: the average of reach variability was $1.44 \pm 0.12^{\circ}$ for the control group and $2.86 \pm 0.23^{\circ}$ for the cerebellar group (mean \pm SEM, two-tailed $t$ test, $p<0.001$ ). This difference in the reach variability was not evident when the visual feedback was removed (two-tailed $t$ test, $p=0.22$ ). Therefore, in the baseline condition, the bias of the movements toward the $45^{\circ}$ target in the cerebellar group was comparable to the control group regardless of visual feedback, though the cerebellar group exhibited greater variability.

We next compared the performance of the two groups in the localization task. This task had two parts. In the first part, subjects used their left hand to point to the remembered location of a visual target (the view of the left hand was always unobstructed). The mean error of the pointing position was $1.36 \pm 0.3^{\circ}$ for the controls and $0.5 \pm 1.02^{\circ}$ for the cerebellar patients (no significant difference; two-tailed $t$ test, $p=0.45$ ). However, the variability of this pointing movement was larger for the cerebellar group (SD of the pointing angle was $0.89 \pm 0.29^{\circ}$ for the control group and $1.44 \pm 0.17^{\circ}$ for the cerebellar group; two-tailed $t$ test, $p<0.05$ ). In the second part, subjects were shown a circle centered on top of their right hand, and then reached to a self-selected position anywhere in the first quadrant. The robot then brought their hand back, and they used their left hand to report where they thought their right hand had crossed the arc. Localization errors as a function of reach angle in the two groups are plotted in Figure $1 D$. We found no systematic differences in the localization performances of the two groups (average localization error, twotailed $t$ test, $p=0.77$ ).

The baseline phase ended with a generalization task in which subjects reached to targets at $45^{\circ}$ and neighboring locations without visual feedback. We observed an interesting trend in the data of both groups: reaches to neighboring targets appeared to be biased toward the $45^{\circ}$ target. For example, small target angles produced positive reach errors, whereas large target angles pro- duced negative reach errors (Fig. $1 E$ ). Statistical analysis showed a main effect of target direction (two-way ANOVA, $F_{(6,102)}=$ $1147, p<0.0001)$. That is, the reaches were attracted to the $45^{\circ}$ target. Interestingly, the bias was stronger in the cerebellar subjects than the controls (group-by-target direction interaction, two-way ANOVA, $\left.F_{(6,102)}=2.95, p=0.01\right)$. Because the baseline phase included repetition of reaches to the $45^{\circ}$ target, the observation of a bias is consistent with the idea that repetition of a reach acts as an attractor, pulling movements of the neighboring directions toward the repeated target (Verstynen and Sabes, 2011). It is possible that in cerebellar patients, repetition produces a larger than normal bias. However, because we did not measure performance in the generalization task before or after repetition of the $45^{\circ}$ target, we cannot determine whether the bias that we are seeing is due to repetition of that movement or due to other factors.

In summary, in the baseline phase of reaching toward the $45^{\circ}$ target, in the baseline phase of the localization task, and in the baseline phase of the generalization task, performances of the two groups were generally comparable in terms of bias, although the cerebellar group tended to show more variability. Repetition of the movements toward the $45^{\circ}$ target affected movements to other targets, resulting in an attraction of the neighboring trajectories. This attraction may have been stronger in cerebellar patients than in healthy controls.

\section{Performance of representative subjects during adaptation}

We used a localization task as a proxy for the forward model before and after adaptation (Fig. 1C). The performances of two representative subjects for two representative movements are shown in the columns labeled "baseline" in Figure 1, F and $G$. The control subject chose to reach to $46^{\circ}$ and then estimated her hand position at $52^{\circ}$. The cerebellar subject chose to reach to $45^{\circ}$ and reported her hand position at $40^{\circ}$. During the adaptation blocks, the subjects were presented with a target at $45^{\circ}$. A perturbation was gradually imposed on the motion of the cursor, rotating it in a counterclockwise direction with respect to motion of the hand. In Figure $1, F$ and $G$, the gray area represents where the hand should move to compensate for the perturbation. Trial after trial, the control and cerebellar subjects altered their motor commands, changing the direction of hand motion. At the end of training, the perturbation reached $+30^{\circ}$. At this stage, in response to the target at $45^{\circ}$, the control subject reached to $\sim 15^{\circ}$ and the cerebellar subject reached to $\sim 17^{\circ}$. After completion of the adaptation phase, we revisited the localization task to assess the state of the forward model. When the control subject reached towards $13^{\circ}$, she localized her hand at $38^{\circ}$, a difference of approximately $+25^{\circ}$ in the direction of the perturbation. In contrast, when the cerebellar subject reached to $\sim 13^{\circ}$, she localized her hand at $22^{\circ}$, a difference of only $+9^{\circ}$. That is, whereas the two subjects appeared to show comparable levels of change in their motor commands during the adaptation block, they showed large differences in their beliefs regarding sensory consequences of these motor commands. The control subject had a much stronger mismatch between actual and reported position of her hand at the end of training than the cerebellar subject did.

\section{Group performance during training}

The behavioral data during adaptation is summarized in Figure 2. Figure $2 \mathrm{~A}$ shows the average reach direction for each group as a function of trial during the adaptation phase. To quantify this change in motor commands, we measured the difference in reach direction between the initial and final five trials (the trials before 
A

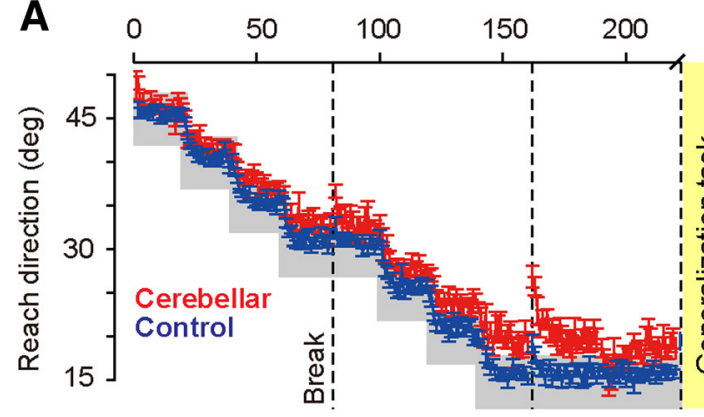

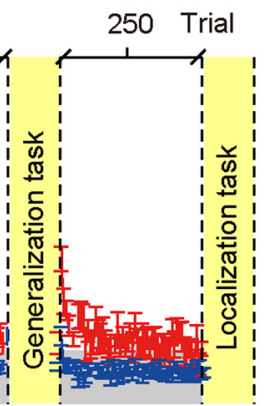

B

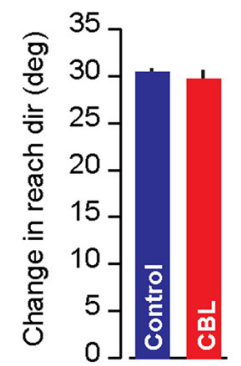

C

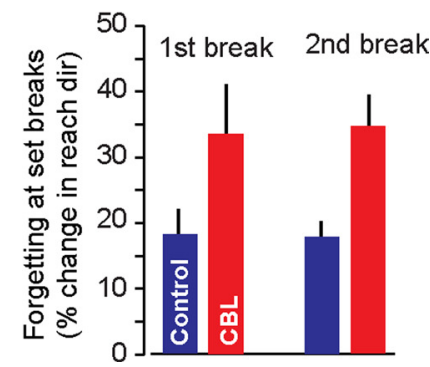

Figure 2. Group performance during training. $A$, Reach direction over trials during the training. The data points are the average ( \pm SEM) of the reach angle across subjects. The gray area indicates reach region for which the cursor would strike the target. $\boldsymbol{B}$, Change in reach angle from the first five trials to the last five trials of training (immediately before generalization task). The two groups displayed comparable change in their motor commands. C, Change in reach angle from the trial before to the trial after the set break. Cerebellar subjects (CBL) exhibited greater forgetting.

the localization task). This change is shown in Figure $2 \mathrm{~B}$. The control subjects showed a change of $30.45^{\circ} \pm 0.37^{\circ}$. The cerebellar subjects showed a change of $29.7^{\circ} \pm 0.94^{\circ}$. This change was comparable in the two groups (two-tailed $t$ test, $p=0.44$ ). These measures were taken at the end of the shooting movement when the hand crossed the target. We also examined performance earlier in the reach at $150 \mathrm{~ms}$ after reach start. The amount of adaptation was again not distinguishable between the two groups (two-tailed $t$ test, $p=0.42$ ). That is, by the end of training in response to the target at $45^{\circ}$, both groups of subjects had learned to alter their motor commands by $\sim 30^{\circ}$.

We noted that during adaptation, the set breaks produced slight forgetting, as performance immediately after the break was worse than immediately before the break. We quantified this forgetting as percentage change in reach angle from the trial before the break to the trial after the break (Fig. 2C). These changes were larger in the cerebellar subjects (repeated-measures ANOVA, main effect of group, $\left.F_{(1,17)}=8.45, p<0.01\right)$, suggesting a larger amount of forgetting due to the set breaks than in controls.

\section{Adaptation of the motor commands}

After 225 adaptation trials, there was another set break, and then we measured performance in a generalization task in which we presented subjects with visual targets at neighboring positions to the trained target. The objective was to quantify the association between the goal of the movement (target position) and the motor commands necessary to achieve that goal, i.e., a proxy for the inverse model. In the generalization block, visual feedback was provided for only the $45^{\circ}$ target and no other targets. In this way, we attempted to maintain the adapted state of the $45^{\circ}$ target (i.e., prevent forgetting) while we measured generalization to other targets. The results are plotted in Figure $3 A$. In Figure 3, the dashed lines represent baseline performance and the solid lines represent performance after adaptation. The shaded area represents the change in the reach angle from baseline to postadaptation. The area of the shaded region is our measure of the total change in the inverse model. This change appeared comparable in the two groups of subjects $\left(1185 \pm 58 \mathrm{deg}^{2}\right.$ in the cerebellar group and $1263 \pm 52 \mathrm{deg}^{2}$ in the control group; two-tailed $t$ test, $p=0.33)$. The change in the reach angles peaked for targets near $45^{\circ}$ (the trained target direction), and then gradually declined for neighboring targets (Fig. 3B). To compare these patterns quantitatively, we fitted a cosine function to the performance of each subject, allowing for a parameter that described location of the peak, a parameter that described magnitude at the peak, and a parameter that described the width of the generalization function. The fitted models are plotted in Figure 3C. While we found no significant difference in the distribution of model parameters for the two groups (two-tailed $t$ test, $p=0.08$ for magnitude of generalization; $p=0.34$ for location of the peak, and $p=0.39$ for the width of the generalization function), there was a trend toward a smaller magnitude of adaptation in the cerebellar subjects, although this trend did not reach significance. Overall, using the area depicted in Figure $3 A$, we would estimate the change in the inverse model for the cerebellar subjects at $93.4 \pm 6 \%$ of that of the control subjects.

\section{Changes in perception of sensory consequences of motor commands}

Following the generalization task, there was another set break, and we then revisited the localization task. As before, without being given an explicit target, the subjects made a movement with their right hand to anywhere along an arc (without visual feedback), the robot brought their hand back to center, and then they used their left hand to report where they thought their right hand had crossed the arc. The results are plotted in Figure 3D. The dashed lines represent baseline performance and the solid lines represent performance in the first five trials after adaptation. The shaded area represents the change in the reach percept from baseline to postadaptation. The area of the shaded region is our proxy for the total change in the forward model. This change was significantly larger in the control group (two-tailed $t$ test, $p<0.0001$ ). Figure $3 E$ is a plot of the change in reach percept with respect to baseline. For control subjects, the training produced a $20^{\circ}$ shift in percept of hand position and was centered on reaching movements toward $30^{\circ}$. For the cerebellar patients, the change in percept was approximately half as much and was centered on reaching movements toward $45^{\circ}$.

Figure $3 E$ is our estimate of the change in the perceived location of the hand as a function of motor commands as measured in the first five trials of the localization task. Because the reach directions were randomly selected by the subjects, the number of data points in each bin was not balanced between groups. Furthermore, using the average of the initial five trials did not allow us to capture the decay properties of this perceptual change. Thus, to quantitatively compare performance in the localization task in the two groups, we fitted a cosine function to the data with parameters that could decay with time (Eq. 1). The left subplot in Figure $3 F$ shows the fitted model's performance for the first localization trial. The parameters that describe the perceptual change were significantly different in the two groups. The peak magnitude was significantly smaller for the cerebellar group (two-tailed $t$ test, $p<0.001$ ), the center was located at a significantly smaller angle for the control group (two-tailed $t$ test, $p=$ 

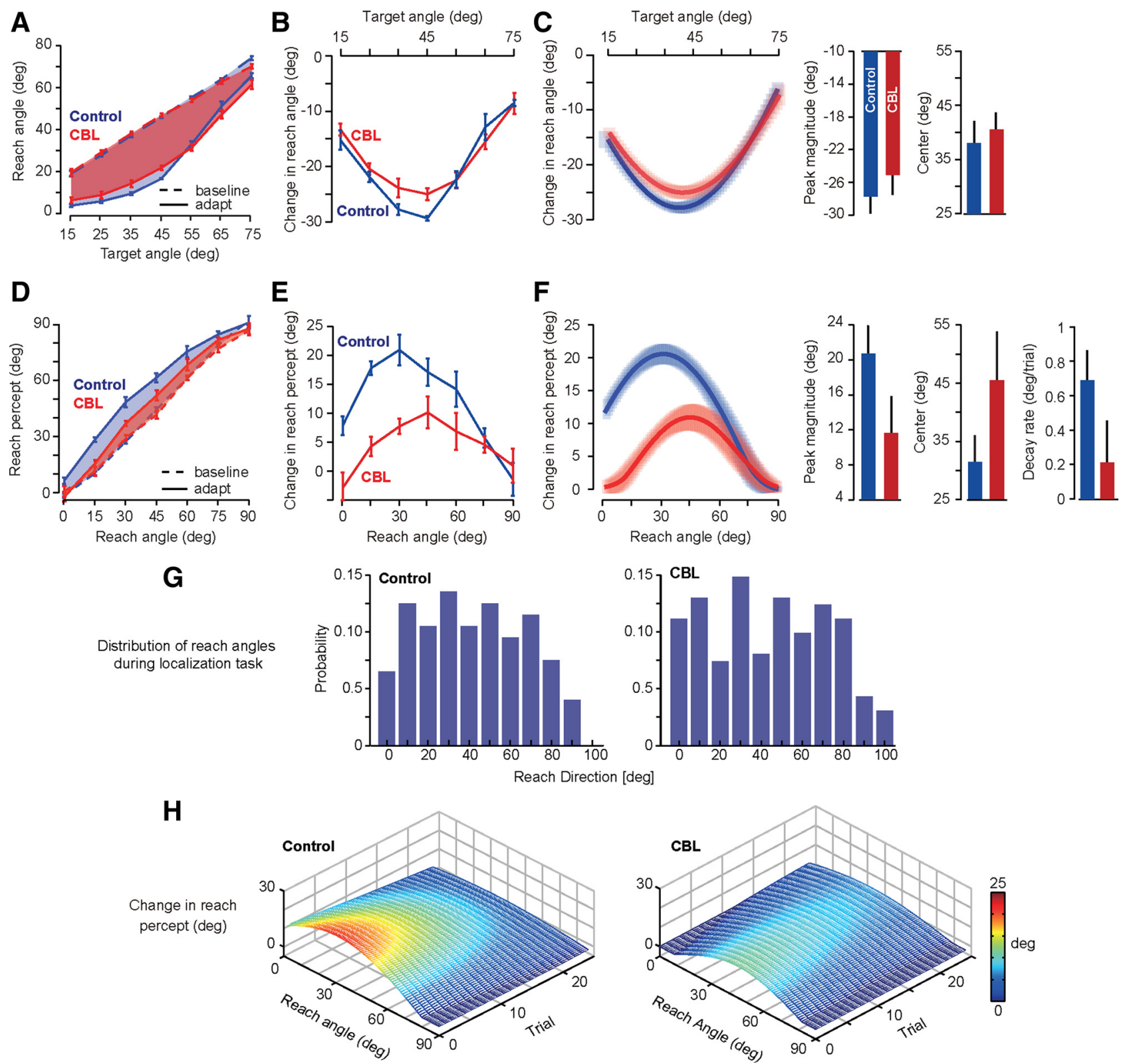

Figure 3. Estimating the change in the inverse and forward models in the control and cerebellar (CBL) groups. $\boldsymbol{A}$, Performance in the generalization task in the baseline (dashed line) and postadaptation (solid line) conditions. The colored regions represent the within-group change in performance. Error bars are SEM. $\boldsymbol{B}$, Within-subject change in reach angle from the baseline to the postadaptation condition in the generalization task (a proxy for the change in the inverse model). Error bars are SEM. C, Results of fitting a cosine function (Eq. 2) to the data in the generalization task. The bar graphs are parameters of the fit. Shaded regions and error bars are $95 \%$ confidence intervals. D, Performance in the localization task in the baseline (dashed line) and postadaptation (solid line, representing the first five trials) conditions. The colored regions are the within-group change in performance. The $y$-axis is the reported reach angle. Error bars are SEM. $E$, Within-subject change in reach percept in the localization task (a proxy for the forward model). Error bars are SEM. $\boldsymbol{F}$, Fit of a cosine function (Eq. 1) to the data in the localization task. The bar graphs (right) are parameters of the fit. The peak magnitude, center location, and decay rate were all significantly different between the two groups. The cosine function (left) represents the change in the forward model, as evaluated at the first localization trial, with respect to baseline. $G$, Distribution of reach directions in the control and cerebellar groups during the localization task. The plot shows the probability of reach angle (bin size, $\left.10^{\circ}\right)$. $\boldsymbol{H}$, Time course of change in reach percept as a function of trial in the postadaptation localization trials. The plots are generated from a fitted model (Eq. 1).

$0.009)$, and the decay rate was significantly smaller for the cerebellar group (two-tailed $t$ test, $p=0.015$ ).

Comparison of the data in the localization task between the control and cerebellar groups is reasonable only if the motor commands produced by the two groups are comparable. Figure $3 G$ plots the distribution of reach directions in the two groups. We found no significant differences in these two distributions (Kolmogorov-Smirnov test, $p=0.86$ ). Figure $3 H$ plots the fitted model of the localization data as a function of reach angle and trial, illustrating that the perceptual change in the control group had a larger initial peak, but also a larger decay rate. Overall, using the area depicted in Figure $3 D$ and the model fits in Figure 3F, we would estimate that perceptual change for the cerebellar subjects was $45 \pm 9 \%$ of that of the control subjects.

\section{Performance of individual cerebellar subjects}

People with cerebellar damage are generally impaired in reach adaptation paradigms, but exhibit between-subject variability that is likely due to the specific location of the cerebellar damage. For example, degeneration of the anterior lobe of the cerebellum affects force field adaptation more than visuomotor adaptation (Rabe et al., 2009; Donchin et al., 2012). Damage in the regions 
served by the SCA impairs adaptation in the force field and visuomotor rotations tasks, but the impairment is less if the damage is to the regions served by the PICA (Donchin et al., 2012). Because we did not have access to MRI data and volumetric analysis, and because our patient population size was small, there is concern that the differences in the localization task may be due to a few patients that may have also learned poorly in the adaptation task.

To consider this question, we plotted a measure of perceptual change as a function of adaptation driven change in motor commands for each subject (Fig. 4A). In Figure $4 A$, the $x$-axis shows an adaptation index for each subject (change in reach direction, end of training with respect to start of training, our measure of change in the inverse model; as in Fig. $2 B$ ) and the $y$-axis shows the average change in reach percept for that subject (our measure of change in the forward model). Along the $x$-axis of Figure $4 A$ there is overlap between the two groups of subjects, reinforcing our observation that the change in motor commands at the end of adaptation was comparable between groups. However, along the $y$-axis we see a strong dissociation between the two groups: the cerebellar group is clustered below the control group. The data in Figure $4 A$ suggests that regardless of how well the cerebellar subjects adapted to the perturbation, they almost uniformly showed a pattern of perceptual change that was smaller than those in healthy controls. The only patient who had a perceptual change within the cluster of healthy controls was Patient 4 . This patient was our most mildly affected individual in terms of clinical symptoms (ICARS score of 5; Table 1).

The between-subject variability in adaptation was greater in the cerebellar subjects than in control subjects (distribution along the $x$-axis; Fig. $4 A$ ). Was some of this variability due to disease severity? In Figure $4 B$, we have plotted change in reach direction at the end of training (with respect to before training) as a function of ataxia score. We found a significant negative correlation $(r=-0.68, p=0.04)$. In Figure $4 C$, we have plotted change in reach direction during the generalization trials (for target at $45^{\circ}$ ) as a function of ataxia score. We again found a significant negative correlation $(r=-0.79, p=$ 0.01 ). In contrast, we found no significant correlation between ataxia score and performance in the localization task $(r=-0.35, p>0.3)$. As the data in Figure $4 \mathrm{~A}$ suggests, when we considered our study groups together, we found a correlation between magnitude of change in the localization task and magnitude of change in motor commands during adaptation training $(r=0.58, p=0.01)$. However, there were no significant correlations within the cerebellar or the control groups.

In summary, despite comparable changes in motor commands in cerebellar and control subjects, the extent of changes in perceived sensory consequences of motor commands in the cerebellar subjects was almost entirely outside the range of healthy controls. The degree of adaptation in motor commands was associated with disease severity.

\section{A potential link between changes in perceived sensory consequences of motor commands and changes in motor commands}

A number of reports have found that a side effect of training in a visuomotor rotation paradigm is a shift in the perceived state of
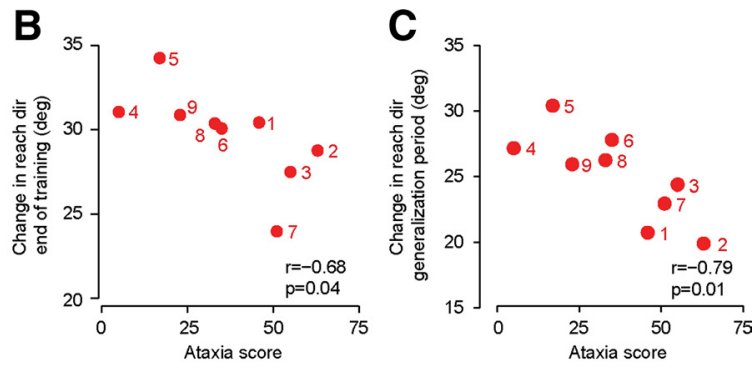

Performance of individual subjects. $\boldsymbol{A}$, Average change in reach percept for each subject, plotted against that subject's from the first five trials to the last five trials of training (immediately before localization task). The $y$-axis is the change in reach cept for each subject (localization task, postadaptation period with respect to preadaptation), averaged over reaches made $15^{\circ}$ and $55^{\circ}$. Blue circles represent healthy controls; red circles represent cerebellar patients. The number next to each (habect five trials of Change in reach angle from the baseline to the postadaptation condition in the generalization task, as measured for reaches to the main target $\left(\right.$ at $\left.45^{\circ}\right)$, is plotted for each subject against that subject's ataxia score.

the arm (Synofzik et al., 2008; Cressman and Henriques, 2009, 2010). Synofzik et al. (2008) interpreted these findings as evidence for a change in a forward model that associates motor commands with sensory consequences. However, Cressman and Henriques (2010) have uncovered evidence arguing that these changes reflect proprioception recalibration and are unrelated to learning an association between motor commands and sensory consequences. Therefore, it is unclear whether changes in perceived state of the arm after visuomotor adaptation are due to changes in forward models, or a form of sensory recalibration. Here we performed a simulation to ask whether the specific pattern of change in percept in the localization task could, in principle, be due to a system that learned to associate motor commands with sensory consequences.

There are two interesting aspects of our data in the control group that are worth highlighting. First, in the localization task in the control group, the peak of the change in percept was not for reaches toward $45^{\circ}$, but $31^{\circ}$. Second, in the generalization task, healthy people generalized their learning in an asymmetric fashion (Fig. $3 B$ ): training to a target at $45^{\circ}$ produced a greater generalization to smaller target angles than larger target angles. Conversations with our colleague Maurice A. Smith raised the idea that both patterns may be a signature of a system that learns to associate motor commands with sensory consequences. We followed up on this theory with a simulation. In our simulation, motor commands on a given trial were associated with a predicted sensory consequence (cursor motion). The difference between observed and predicted consequences produced adaptation in the forward model. On the next trial, the motor command that was generated was based on a search of the possible commands and their expected sensory consequences (Eq. 6). In this way, changes in the forward model directly impacted both the percept about sensory consequences and the motor commands on the next trial. We show that such a system can, in principle, account for the asymmetry in generalization (Fig. 3B) and the pattern of change in percept (Fig. 3E).

The simulation results are shown in Figure 5. With each step in the perturbation, the reach direction changes to bring the cursor close to the target at $45^{\circ}$ (Fig. $5 A$ ). By end of training, in response to the $45^{\circ}$ target, the motor commands are reaching toward $15^{\circ}$, a change of $-30^{\circ}$ from baseline. We have plotted the state of the forward model during training as would be estimated 
A

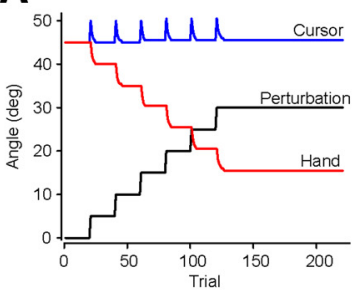

B
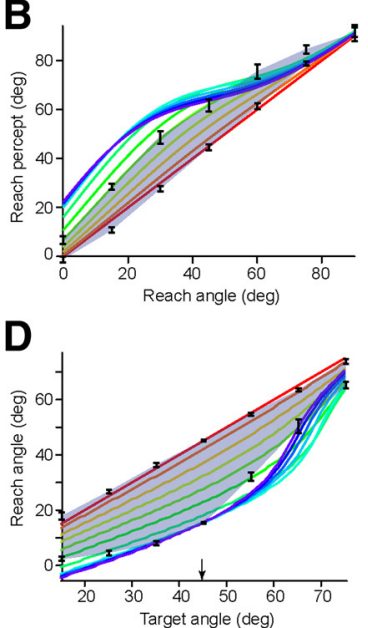

C

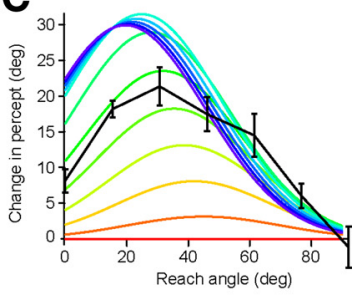

E

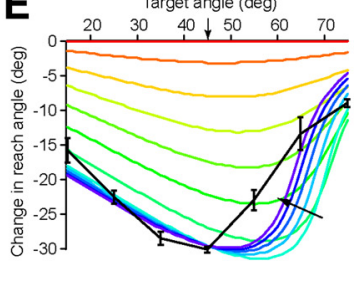

Figure 5. Simulation results. $\boldsymbol{A}$, The simulated adaptive system was trained on a perturbation protocol similar to the one experienced by the subjects. $\boldsymbol{B}$, State of the forward model in trials $1,21,41$, etc. Red line is the state at baseline; dark blue line is the state at end of training. With increased trials, the peak shifts to smaller reach angles. Superimposed on the simulation data are data from control subjects in the localization task (Fig. 3D). C, Change in the forward model with respect to baseline. The black line is data from control subjects in the localization task (Fig. 3E). D, Reach angle as a function of target direction. The colored lines represent state of the inverse model (Eq. 6) in trials 1, 21,41, etc. Red line is the state at baseline; dark blue line is the state at end of training. With increased trials, the generalization narrows for larger target angles. Superimposed on the simulation data are data from control subjects in the generalization task (Fig. $3 A$ ). Arrow indicates the trained target direction $\left(45^{\circ}\right)$. $\boldsymbol{E}$, Change in the inverse model with respect to baseline. The black line is data from control subjects (Fig. 3B).

in the localization task at intervals of 20 trials in Figure $5, B$ and $C$. The effect of training is a shift in the peak of the forward model from reach angles at $45^{\circ}$ to $\sim 20^{\circ}$. This is a reflection of the history of motor commands and sensory prediction errors during training. Superimposed on this plot is the data from the localization task in control subjects (Fig. 3D,E). The control subjects exhibited a maximum change in their percept of hand position for reach angles at $\sim 31^{\circ}$, whereas cerebellar subjects exhibited a peak at $45^{\circ}$. The bias of the data in the control group toward smaller reach angles is consistent with the hypothesis that the localization task is measuring a component of the forward model. However, the magnitude of the peak and its shift in location are smaller in the measured data than predicted by the simulations.

Now let us consider the interesting fact that the generalization function (Fig. 3B) is asymmetric. We can show that this may be linked to adaptation of the forward model. In our simulations, the forward model is represented by a set of basis functions that encodes the map from motor commands to motion of the arm (reach angle). To generate the motor commands, our simulations relied on these bases to predict the sensory consequences, and selected those commands that brought the arm to the target location. Therefore, the representation that we chose for simulating the forward model directly predicts what we should see during the generalization task, i.e., our proxy for the inverse model. In Figure 5, $D$ and $E$, we have plotted the state of the inverse model during training as would be measured in the generalization task at intervals of 20 trials. Superimposed on this plot is the data from the generalization task in control subjects (Fig. $3 B, C$ ). With increased training, the generalization of the simulation narrows for larger target angles but remains unchanged for smaller target angles. As a result, in our simulations, the generalization profile of the inverse model at the end of training is asymmetric, skewed toward smaller target angles (Fig. 5E). It is interesting that the control group also exhibited greater generalization toward smaller target angles than larger angles (two-tailed $t$ test, $p<0.05$;
Fig. $3 B$, slope of the left and right sides of the generalization function). According to the simulations, the reason for this asymmetry is that the motor commands on each trial are relying on a forward model that associates previous motor commands with their sensory consequences. The generalization data for the cerebellar group exhibited no significant asymmetry (two-tailed $t$ test, $p=0.10$; Fig. 3B, slope of the left and right sides), although comparison of these two groups did not reach significance (two-tailed $t$ test, $p=0.55$; Fig. $3 B$, difference in slope of left and right sides).

In summary, we performed a simulation in which, on a given trial, our system used its forward model to search for the best motor command that would produce a sensory consequence consistent with moving a cursor to a target. Once the motor command was generated, it predicted the sensory consequences of it, and then learned from the difference between the predicted and actually observed sensory consequences. This updating of the forward model affected the motor commands on the next trial. Our simulation predicted two interesting results. First, in the localization task, the peak of the change in percept should not be for reaches to the trained target, but for reaches toward targets that are at smaller angles. Second, in the generalization task, motor commands should show asymmetric generalization, displaying greater generalization to angles that are smaller than the trained target. We observed both of these features in the data of our healthy controls.

In contrast, a feature of the data that our model could not account for was the lack of correlation between degree of change in the localization task and degree of change in the motor commands. As is evident in Figure 4A, in healthy individuals, there is no correlation between change in percept in the localization task and amount of adaptation in motor commands during training. Our simulations predicted existence of this correlation because change in the forward model is the only signal that produces change in the motor commands. Recent results have demonstrated that error-dependent learning (which in our simulations drives the change in the forward and inverse models) is only one component of motor learning and there exists other components that depend on reward prediction error (Izawa and Shadmehr, 2011; Pekny et al., 2011).

\section{Discussion}

Let us define an inverse model as a map that associates goal of the movement (e.g., placing a cursor in a target position) with motor commands that achieve that goal, and define a forward model as a map that associates motor commands with their sensory consequences. Does damage to the cerebellum affect learning of inverse models, forward models, or both? To produce learning in these hypothetical maps, we perturbed the relationship between displacement of the hand and displacement of the cursor. The training focused on a single target position. To assay the change in the inverse model, we presented people with goals near the trained target. We observed that people with cerebellar damage learned to alter their motor commands in response to the trained 
target and generalized this to neighboring targets in a way that was similar to healthy controls. To assay the change in the forward model, people self-selected a movement without an explicit target and then reported where they believed their hand had moved to (Synofzik et al., 2008). In healthy people, the training induced significantly larger shifts in predicted sensory consequences of motor commands than in people with cerebellar damage.

Numerous studies have documented cerebellar involvement in motor adaptation. Yet, the computational function of the cerebellum during motor adaptation remains unclear. Some have suggested that the function of the cerebellum is to act as an inverse model, generating the sequence of the motor commands that take into account dynamics of the body (Gomi and Kawato, 1992; Kawato and Gomi, 1992a,b). Some have suggested that the function of the cerebellar is to act as a forward model, predicting the sensory consequence of action (Wolpert and Miall, 1996; Doya, 1999; Paulin, 2005; Shadmehr and Krakauer, 2008; Izawa and Shadmehr, 2011). Still others have suggested a function that involves both kinds of internal models (Wolpert and Kawato, 1998).

The results from our localization task support the hypothesis that the cerebellar function during motor learning is related to formation of forward models. Our simulations showed that as a consequence of adaptation, the peak of the change in the forward model should have shifted from a reach angle at $45^{\circ}$ (target direction) to $\sim 20^{\circ}$. This view is consistent with the results of Gonzalez Castro et al. (2011), who observed that during reach adaptation in force fields, adaptation was greatest along sensory states that were most repeated, and not along the target directions that were most repeated. As a consequence, our simulations predicted that the maximum illusion in the localization task caused by the training in the reaching task should have been for reaches $25^{\circ}$ away from the trained target direction. In control subjects, we observed a shift of $14^{\circ}$, but observed no shift in the cerebellar group. Furthermore, the simulations predicted that the peak change in the localization task should have a magnitude of $\sim 30^{\circ}$. In control subjects, we observed a magnitude of $21^{\circ}$, and in the cerebellar group the magnitude was $11^{\circ}$.

\section{Limitations of the localization task as an assay of the forward model}

There are two kinds of sensory consequences associated with motor commands: visual feedback associated with the cursor and proprioceptive feedback associated with the arm. Furthermore, when one is holding a novel tool, there are two kinds of possible perturbations: the tool can act as a perturbation that alters both forms of sensory feedback and the visual display can act as a perturbation that alters only the visual feedback. In this scenario, Haith et al. (2008) showed theoretically that some fraction of the prediction error is assigned to a perturbation to the arm, and some to the visual display of hand position (Shadmehr and Mussa-Ivaldi, 2012). The localization task assays the net change in proprioceptive state of the arm, but does not assay the change caused by the perturbation to the visual feedback (the cursor). Therefore, from a theoretical perspective, the localization task provides only a partial picture of the change in the forward model. Furthermore, as Cressman and Henriques (2010) have demonstrated, changes in perceived location of the arm occur even when one passively views movements of the hand in a visuomotor rotation paradigm. Although the effects reported by that study are threefold smaller than what we see here, it suggests that changes in percept cannot be viewed solely as a signature of a system that associates motor commands with sensory consequences. Rather, the changes that we recorded in perception of arm position may be due to a combined change in forward model and in association of proprioception with vision. Importantly, we observed that the peak change in percept in the patients was centered at the trained target direction, whereas the peak in the controls was shifted. Our simulations suggest that this shift is a signature of adaptation in a map that associated motor commands with sensory consequences. It is possible that what we observed in cerebellar patients is a relatively intact adaptation of maps that associate one sensory modality with another, but impaired adaptation in maps that associate motor commands with sensory consequences. In support of this theory, a recent experiment in some of the same patients that we studied found normal sensory realignment in a task in which proprioceptive state of the arm was matched with a visual cursor (H. Block and A. Bastian, unpublished observations).

\section{Learning from sensory prediction error to alter motor commands}

Does adaptation in a forward model contribute to changes in motor commands? In our simulation we assumed that the motor commands are generated through a search of the motor space, finding the best command in terms of a match between expected sensory consequences and target. Partial evidence in support of this idea is that the simulations predicted an asymmetric generalization profile, something that agreed with our observations (Fig. 5E). However, recent studies of motor learning have observed evidence of a specialized inverse model that can be learned independently of the forward model through reinforcement learning (Izawa and Shadmehr, 2011) or repetition (Diedrichsen et al., 2010; Huang et al., 2011; Verstynen and Sabes, 2011). Furthermore, in our healthy subjects, we did not observe a correlation between magnitude of change in the localization task (proxy for the forward model) and magnitude of change in the reach direction (proxy for the inverse model) (Fig. 4A), as might be expected if changes in the forward model are the only factor that drive changes in the inverse model. Therefore, it seems likely that changes that take place in motor commands during adaptation are only partly driven by changes in forward models.

\section{Learning despite a damaged cerebellum}

Our results strengthen our previous report in a force field adaptation paradigm: people with cerebellar damage maintain an ability to improve their motor commands in response to a gradually increasing perturbation (Criscimagna-Hemminger et al., 2010). It is possible that the cerebellar patients may have adapted by relying on reward prediction error. When adaptation is driven solely by reward prediction error, the resulting behavior has two signatures: little or no change in the forward model as assayed in the localization task, and a narrow generalization function as assayed in the reach to target task (Izawa and Shadmehr, 2011). In contrast, our cerebellar patients exhibited smaller than normal changes in the localization task, but a reach to target generalization function that was comparable to healthy people (Fig. $3 B$ ). Therefore, performance of cerebellar patients is not consistent with the hypothesis that, in the gradual paradigm, they relied solely on reward prediction errors.

Because cerebellar degeneration is a chronic disease, the brain may engage neural mechanisms that allow for partial compensation. A potential clue is that both here and in an earlier reaching experiment (Criscimagna-Hemminger et al., 2010), we found that cerebellar patients showed a greater amount of forgetting at 
set breaks [however, note that in saccade adaptation, cerebellar subjects exhibit little or no forgetting (Xu-Wilson et al., 2009)]. This suggests that in reach adaptation in cerebellar patients, there is a stronger than normal dependence on a fast process (Smith et al., 2006), a process that shares resources with the declarative system (Keisler and Shadmehr, 2010). It is possible that the ability of the cerebellar patients to adapt in the gradual condition is partly due to a greater than normal reliance on the declarative system. Two points, however, argue against this idea. First, we observed normal generalization in our assay of the inverse model. Second, we observed slower than normal decay in the assay of the forward model in the localization task. More studies are needed to understand the neural mechanisms that allow cerebellar patients to improve their performance in the gradual perturbation schedule.

In summary, we found that despite cerebellar damage, the brain maintained an ability to learn motor commands that effectively canceled the perturbation in a reaching task in which the perturbation was imposed gradually. However, cerebellar damage impaired the ability to learn the visual sensory consequences of those commands.

\section{References}

Cressman EK, Henriques DY (2009) Sensory recalibration of hand position following visuomotor adaptation. J Neurophysiol 102:3505-3518.

Cressman EK, Henriques DY (2010) Reach adaptation and proprioceptive recalibration following exposure to misaligned sensory input. J Neurophysiol 103:1888-1895.

Criscimagna-Hemminger SE, Bastian AJ, Shadmehr R (2010) Size of error affects cerebellar contributions to motor learning. J Neurophysiol 103:2275-2284.

Diedrichsen J, Hashambhoy Y, Rane T, Shadmehr R (2005) Neural correlates of reach errors. J Neurosci 25:9919-9931.

Diedrichsen J, White O, Newman D, Lally N (2010) Use-dependent and error-based learning of motor behaviors. J Neurosci 30:5159-5166.

Donchin O, Rabe K, Diedrichsen J, Lally N, Schoch B, Gizewski ER, Timmann D (2012) Cerebellar regions involved in adaptation to force field and visuomotor perturbation. J Neurophysiol 107:134-147.

Doya K (1999) What are the computations of the cerebellum, the basal ganglia and the cerebral cortex? Neural Netw 12:961-974.

Gomi H, Kawato M (1992) Adaptive feedback control models of the vestibulocerebellum and spinocerebellum. Biol Cybern 68:105-114.

Gonzalez Castro LN, Monsen CB, Smith MA (2011) The binding of learning to action in motor adaptation. Plos Comp Biol 7:e1002052.

Haith A, Jackson C, Miall C, Vijayakumar S (2008) Interactions between sensory and motor components of adaptation predicted by a Bayesian model. In: Advances in Computational Motor Control Washington, DC.

Haruno M, Wolpert DM, Kawato M (2001) Mosaic model for sensorimotor learning and control. Neural Comput 13:2201-2220.

Huang VS, Haith A, Mazzoni P, Krakauer JW (2011) Rethinking motor learning and savings in adaptation paradigms: model-free memory for successful actions combines with internal models. Neuron 70:787-801.

Izawa J, Shadmehr R (2008) On-line processing of uncertain information in visuomotor control. J Neurosci 28:11360-11368.

Izawa J, Shadmehr R (2011) Learning from sensory and reward prediction errors during motor adaptation. PLoS Comput Biol 7:e1002012.

Kawano K, Shidara M, Takemura A, Inoue Y, Gomi H, Kawato M (1996) Inverse-dynamics representation of eye movements by cerebellar Purkinje cell activity during short-latency ocular-following responses. Ann N Y Acad Sci 781:314-321.
Kawato M (1999) Internal models for motor control and trajectory planning. Curr Opin Neurobiol 9:718-727.

Kawato M, Gomi H (1992a) The cerebellum and VOR/OKR learning models. Trends Neurosci 15:445-453.

Kawato M, Gomi H (1992b) A computational model of four regions of the cerebellum based on feedback-error learning. Biol Cybern 68:95-103.

Keisler A, Shadmehr R (2010) A shared resource between declarative memory and motor memory. J Neurosci 30:14817-14823.

Körding KP, Wolpert DM (2004) Bayesian integration in sensorimotor learning. Nature 427:244-247.

Maschke M, Gomez CM, Ebner TJ, Konczak J (2004) Hereditary cerebellar ataxia progressively impairs force adaptation during goal-directed arm movements. J Neurophysiol 91:230-238.

Miall RC, Christensen LO, Cain O, Stanley J (2007) Disruption of state estimation in the human lateral cerebellum. PLoS Biol 5:e316.

Nowak DA, Topka H, Timmann D, Boecker H, Hermsdörfer J (2007) The role of the cerebellum for predictive control of grasping. Cerebellum $6: 7-17$.

Paulin MG (2005) Evolution of the cerebellum as a neuronal machine for Bayesian state estimation. J Neural Eng 2:S219-S234.

Pekny SE, Criscimagna-Hemminger SE, Shadmehr R (2011) Protection and expression of human motor memories. J Neurosci 31:13829-13839.

Rabe K, Livne O, Gizewski ER, Aurich V, Beck A, Timmann D, Donchin O (2009) Adaptation to visuomotor rotation and force field perturbation is correlated to different brain areas in patients with cerebellar degeneration. J Neurophysiol 101:1961-1971.

Shadmehr R, Krakauer JW (2008) A computational neuroanatomy for motor control. Exp Brain Res 185:359-381.

Shadmehr R, Mussa-Ivaldi S (2012) Biological learning and control: how the brain builds representations, predicts events, and makes decisions. Cambridge, MA: MIT.

Shadmehr R, Smith MA, Krakauer JW (2010) Error correction, sensory prediction, and adaptation in motor control. Annu Rev Neurosci 33:89-108.

Smith MA, Shadmehr R (2005) Intact ability to learn internal models of arm dynamics in Huntington's disease but not cerebellar degeneration. J Neurophysiol 93:2809-2821.

Smith MA, Ghazizadeh A, Shadmehr R (2006) Interacting adaptive processes with different timescales underlie short-term motor learning. PLoS Biol 4:e179.

Synofzik M, Lindner A, Thier P (2008) The cerebellum updates predictions about the visual consequences of one's behavior. Curr Biol 18:814-818.

Trouillas P, Takayanagi T, Hallett M, Currier RD, Subramony SH, Wessel K, Bryer A, Diener HC, Massaquoi S, Gomez CM, Coutinho P, Ben Hamida M, Campanella G, Filla A, Schut L, Timann D, Honnorat J, Nighoghossian N, Manyam B (1997) International Cooperative Ataxia Rating Scale for pharmacological assessment of the cerebellar syndrome. The Ataxia Neuropharmacology Committee of the World Federation of Neurology. J Neurol Sci 145:205-211.

Tseng YW, Diedrichsen J, Krakauer JW, Shadmehr R, Bastian AJ (2007) Sensory prediction errors drive cerebellum-dependent adaptation of reaching. J Neurophysiol 98:54-62.

Vaziri S, Diedrichsen J, Shadmehr R (2006) Why does the brain predict sensory consequences of oculomotor commands? Optimal integration of the predicted and the actual sensory feedback. J Neurosci 26:4188-4197.

Verstynen T, Sabes PN (2011) How each movement changes the next: an experimental and theoretical study of fast adaptive priors in reaching. J Neurosci 31:10050-10059.

Wolpert DM, Kawato M (1998) Multiple paired forward and inverse models for motor control. Neural Netw 11:1317-1329.

Wolpert DM, Miall RC (1996) Forward models for physiological motor control. Neural Netw 9:1265-1279.

Xu-Wilson M, Zee DS, Shadmehr R (2009) The intrinsic value of visual information affects saccade velocities. Exp Brain Res 196:475-481. 